\title{
Multiple Abdominal Masses: Report of an Unusual Case
}

\author{
Vijay Ramachandran ${ }^{*}, 1$ and Krishnaprasad Patla ${ }^{2}$ \\ ${ }^{I}$ Department of Surgical Gastroenterology, Yenepoya Medical College \& Hospital, Deralakatte, Mangalore, India \\ ${ }^{2}$ Department of Anaesthesiology, K.S. Hegde Medical Academy, Deralakatte, Mangalore, India
}

\begin{abstract}
Patients presenting with multiple abdominal masses is quite unusual. The usual differential diagnoses entertained include Tuberculosis and Lymphoma. However, occasionally, the verdict delivered by the pathologist is a surprise. We report the successful resection of three intraabdominal masses which revealed a unique histopathological entity.
\end{abstract}

Keywords: Abdominal mass, Desmoplastic, Round cell tumour.

\section{INTRODUCTION}

Desmoplastic small round cell tumor is a rare malignancy with poor prognosis that predominantly affects young males. Its etiopathogenesis is still unknown and diagnosis can be achieved only by immunohistochemistry and cytogenetic studies. Due to our limited knowledge of the pathologic and clinical nature of this disease, there is no clear consensus regarding the optimal therapeutic procedures for treating this neoplasm. A high degree of care and improvements in diagnostic capabilities are required in order to identify this entity and avoid misdiagnosis.

\section{CASE REPORT}

A 40 year old man presented with persistent epigastric pain and mass per abdomen of one month duration. The patient denied history of vomiting, anorexia, weight loss, night sweats, melaena or altered bowel habits. Clinical examination revealed a mass with restricted mobility, $10 \times 6$ $\mathrm{cms}$ in size, in the epigastrium extending to the periumbilical region and another mass $5 \times 4 \mathrm{cms}$ in the Right Iliac Fossa. Digital Rectal examination revealed an extrinsic mass $8 \mathrm{cms}$ from the anal verge. He was initially evaluated with routine blood tests, peripheral smear and an Upper G.I. Endoscopy (UGIE) which were normal. CECT abdomen (Fig. 1) revealed the presence of a mass $10 \times 6 \mathrm{cms}$ in size, enhancing well with contrast, located between the stomach and the transverse colon. In addition, two other masses, one in the ileal mesentery and another in the lateral wall of the lower rectum each $5 \times 4 \mathrm{cms}$ in size were noted. As the masses appeared resectable on CT Scan, he was taken up for surgery. Operative findings included $10 \times 6 \mathrm{cms}$ mass, infiltrating the stomach, abutting the transverse colon (Fig. 2) and masses in the ileal mesentery and lateral rectal wall as described earlier. There were subcentimeter deposits in the diaphragmatic peritoneum and the lesser omentum as well. Resection of the masses with negative margins and sleeve

\footnotetext{
*Address correspondence to this author at the Department of Surgical Gastroenterology \& Minimal Access Surgery, Yenepoya Medical College, Deralakatte, Mangalore, Karnataka, PIN - 575018, India; Tel: +91 824 2432916, +91 97312 31769; Fax: 0824 2496800;

E-mail: drvijayr@gmail.com
}

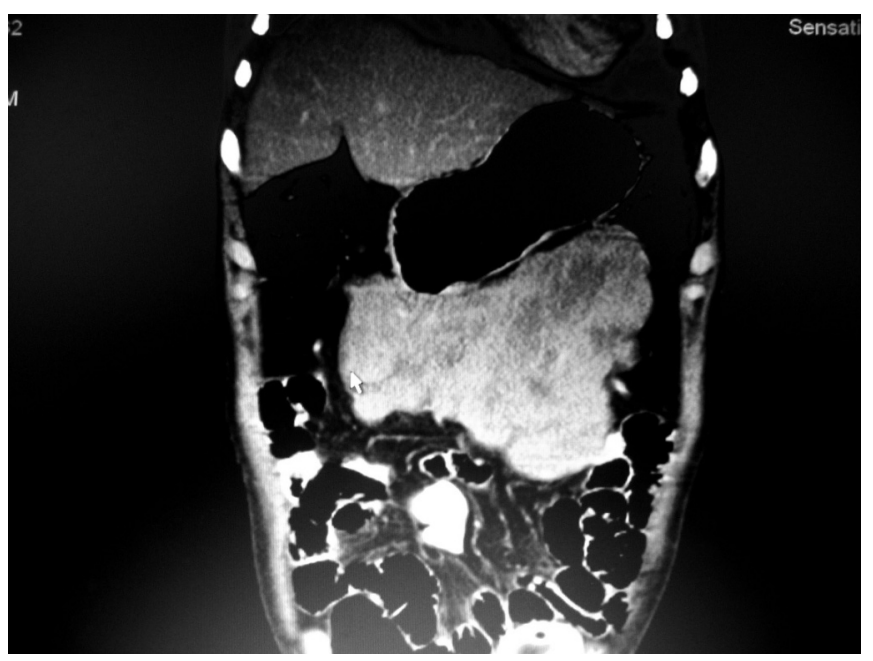

Fig. (1). Axial contrast enhanced CT image shows a hyperdense mass with hypodense areas in the vicinity of stomach and transverse colon.

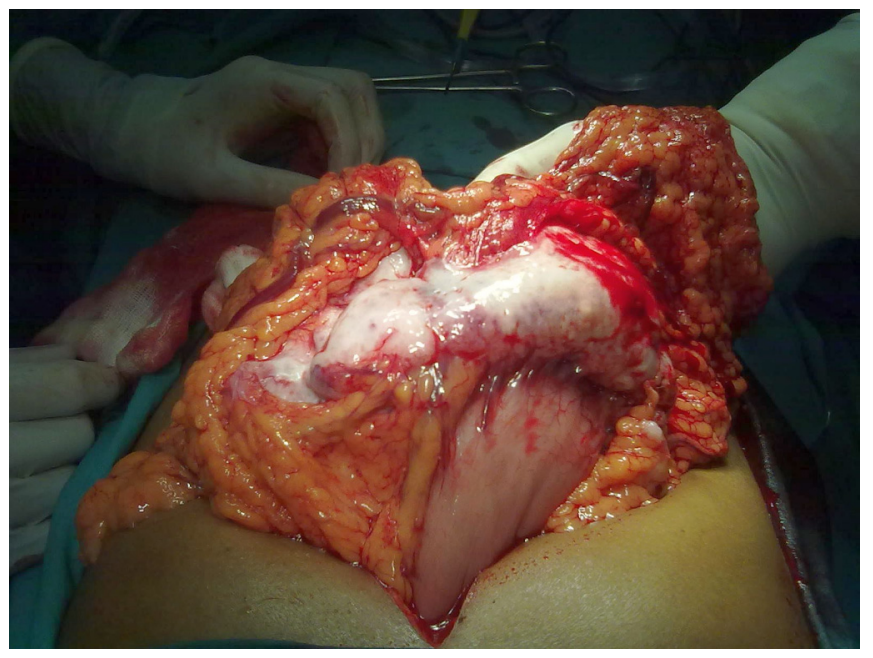

Fig. (2). Peroperative photograph showing the hard mass arising from the mesentery and infiltrating the stomach.

gastrectomy was done. Patient developed vomiting in the postoperative period which responded to prokinetics and was 
subsequently discharged. Two weeks following discharge, he was readmitted with symptoms of persistent vomiting and was subjected to an UGIE which did not reveal any narrowing of the gastric lumen. Patient improved after the procedure and administration of prokinetics and was discharged. Histopathology of the resected specimen revealed nests of small tumour cells with hyperchromatic nuclei and scanty cytoplasm in a desmoplastic stroma (Fig. 3). Immunohistochemistry was negative for S-100, Neuron Specific Enolase, Synaptophysin and Chromogranin and was positive for Desmin, Vimentin and Cytokeratin. The resected margins were free of tumour. The features were typical of Desmoplastic Small Round Cell Tumour (DSRCT). Owing to the disseminated nature of the disease, he was started on combination chemotherapy.

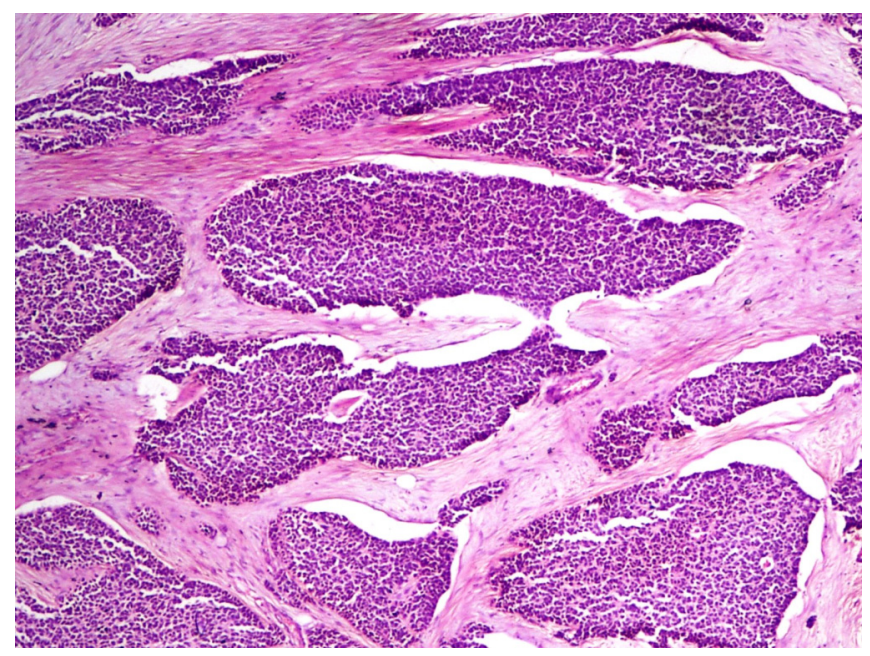

Fig. (3). Nests of small tumour cells, with scanty cytoplasm and dark, hyperchromatic nuclei and a few rossettes are seen. The stroma shows desmoplasia.

He is presently asymptomatic at 3 months follow up.

\section{DISCUSSION}

DSRCT belongs to the family of "Small Round Blue Cell Tumours" of the paediatric population and was first described by Gerald and Rosai in 1989 [1]. It typically afflicts young males and presents as an abdominal mass [2]. DSRCT is associated with a characteristic balanced translocation between chromosomes $11 \& 22$, similar to Ewing's Sarcoma [3]. These tumours purportedly arise from mesothelial cells and have been decribed at a variety of sites including the paratesticular region, pleura, lung, ovary, sinus cavity, central nervous system and stomach [4]. CT Scan aids in the diagnosis of these tumours by demonstrating multiple, low attenuation soft tissue masses in the omentum or mesentery without a distinct organ of origin. Despite the multiplicity of tumours, the lesion is considered as multifocal rather than metastatic at presentation [5]. The usual differential diagnoses include Rhabdomyosarcoma, Tuberculosis, Lymphoma, Carcinoids, Malignant Fibrous Histiocytoma,
Neuroblastoma, Mesothelioma, Peritoneal leiomyosarcomatosis, Intraabdominal desmoids, Fibrosing mesenteritis, Splenosis and Amyloidosis. Immunohistochemistry is usually positive for Desmin, Vimentin, Cytokeratin, Epithelial Membrane Antigen and Placental Alkaline Phosphatase. Response to Trastuzumab and Imatinib Mesylate depends on expression of Her-2 neu receptor and Platelet Derived Growth Factor Receptor in these tumours. Despite aggressive treatment including Surgical debulking, Radio-therapy and Multiagent Chemotherapy, the prognosis in DSRCT is poor and the mean survival is 17 months [6]. Newer treatment modalities include hyperthermic intraperitoneal chemotherapy using cisplatin \& Yttrium microspheres for liver metastases [7]. Analysis of one of the largest series of 40 cases of DSRCT has revealed that the overall survival is $29 \%$ at 3 years. Induction chemotherapy (P6) followed aggressive surgical debulking and external beam radiotherapy has been recommended for the treatment of these tumours [8].

\section{CONCLUSION}

This case report highlights a unique pathological diagnosis in a patient who presented with symptoms of a lump in the abdomen and was noted to have multiple soft tissue density masses. The need to include Desmoplastic Small Round Cell Tumour (DSRCT) in the list of differential diagnoses in such a setting cannot be overemphasised.

\section{ACKNOWLEDGMENT}

None declared.

\section{CONFLICT OF INTEREST}

None declared.

\section{REFERENCES}

[1] Gerald WL, Rosai J. Case 2. Desmoplastic small cell tumour with divergent differentiation. Paediatr Pathol 1989; 9: 177-83.

[2] Dorsey BV, Benjamin LE, Rauscher F, et al. Intraabdominal desmoplastic small round cell tumour: expansion of the pathologic profile. Mod Pathol 1996; 9: 703-9.

[3] Gerald WL, Ladanyi M, de Alava E, et al. Clinical, pathologic and molecular spectrum of tumours associated with $\mathrm{t}(11 ; 22)(\mathrm{p} 13 ; \mathrm{q} 12)$ : desmoplastic small round cell tumour and its variants. J Clin Oncol 1998; 16: 3028-36.

[4] Lippe P, Berardi R, Cappelletti C, et al. Desmoplastic small round cell tumour: Description of two cases and review of the literature. Oncology 2003; 64: 14-7.

[5] Hayes- Jordan A, Anderson PM. The diagnosis and management of desmoplastic small round cell tumour. A Review. Curr Opin Oncol 2011; 23(4): 385-9.

[6] Biswas G, Laskar S, Banavali SD, et al. Desmoplastic small round cell tumour: A description of two cases and review of the literature. Indian J Cancer 2005; 42: 78-84.

[7] Subbiah V, Murthy R, Anderson PM. (90Y)Yttrium microspheres radioembolotherapy in desmoplastic small round cell tumour hepatic metastases. J Clin Oncol 2011; 29(11): e292-4.

[8] Quaglia MP, Brennan MF. The clinical approach to desmoplastic small round cell tumour. Surg Oncol 2000; 9(2): 77-81. 